Scientific Visualization, 2020, volume 12, number 2, pages 74 - 83, DOI: 10.26583/sv.12.2.06

\title{
Visualization of whirlwind (non-stationary vortex) structures aimed to the improvement of cooling systems of electric power devices
}

\author{
A.Yu. Varaksin',A, K.K. Denshchikov²,B, M.V. Protasov3,B, M.E. Romash4,B \\ A Bauman Moscow State Technical University \\ B Joint Institute for High Temperatures, Russian Academy of Sciences, Russia \\ ${ }^{1}$ ORCID: 00oo-0002-8799-6378, varaksin a@mail.ru \\ 2 ORCID: 0000-0002-9256-1491, kdenshchikov@mail.ru \\ 3 ORCID: 0000-0003-0711-9437, protasov m@mail.ru \\ 4 ORCID: 0000-0002-3524-078X, romash661@mail.ru
}

\begin{abstract}
This paper concerns with the problems of cooling down of various high-voltage electric power devices of the megawatt range. When flowing around the current-carrying and other elements of these devices a formation of non-stationary vortices can take place. Therefore, a detection and visualization of vortex structures appearing at the coolant flowing through channels with the complicated geometry is an important task.

The generation of whirlwind structures (non-stationary vortices) was performed above this substrate surface (aluminum alloy sheet) by the unstable air stratification obtained by heating it up from below. We have used the mcm sized particles of magnesia deposited on the substrate surface to visualize the generated free non-stationary vortices.

The frame-by-frame view of recordings obtained by the direct video-registration at various thermal modes allowed performing of the qualitative analysis of the spatio-temporal structure of non-stationary vortices and obtaining a lot of their important integral parameters (visible height, diameter, lifetime, quantity of vortices, base motion velocity, etc.).

There had been considered physical basements of processing of flow patterns obtained by the PIV method with the use of the measurer of fields of velocities POLIS (Russian "ПОЛИС", the instrument is developed by the S.S. Kutateladze Institute for Thermophysics of the Siberian Branch of RAS, Novosibirsk) in order to recover the fields of instantaneous velocities of vortex structures.

The results of the visualization of free non-stationary vortices obtained by the direct video filming and vector fields of instantaneous velocities, obtained by the PIV (Particle image velocimetry) method, have been presented.

The developed technique of visualization and diagnosis of free non-stationary vortices can be used for development of the state-of-the-art cooling systems of high-power electric devices.
\end{abstract}

Keywords: visualization, free non-stationary vortices, cooling down of electric power devices, PIV method, vector field of velocities.

\section{Introduction}

One of the most important tasks for the development of the state-of-the-art electric power devices and systems is the problem of removal of highly intensive heat fluxes [1-3]. In electric power systems operating at high voltages $(>1 \mathrm{kV})$ and powers $(>1 \mathrm{MWt})$ the heat flux intensity can reach Wt/cm $\mathrm{cm}^{2}\left(1 \mathrm{MWt} / \mathrm{m}^{2}\right)$. The designers of high-power electric equipment have to solve an uncommon task how to enhance the cooling down efficiency at the 
high hydraulic resistance and increasing of coolant transport power. There have been proposed various solutions of this problem: 1) heat transfer intensification with the use of turbulent flow modes of cooling gases; 2) using of dielectric liquids (e.g., Novec 649) [4], with the boiling temperature lower than one of the heat generating elements surface, etc.

Turbulent flow modes are characterized by the presence of vortex structures having various sizes. It should be noted that non-stationary vortices can be formed when passing over the details of electric power devices. Therefore, the visualization and diagnosis of vortex structures, appearing at the coolant flow via channels with complicated configuration is an important task.

This work is aimed to the visualization of free non-stationary vortices by direct video filming and measurement of vector fields of the instantaneous velocities within the vortex transversal cross-section area with use of the PIV method.

\section{Experimental unit to generate free non-stationary vortices}

The generation of vortex structures was performed by the creation of the unstable air stratification above the substrate surface of an aluminum sheet (1100 $\mathrm{mm}$ of diameter and 1.5 $\mathrm{mm}$ of thickness), heated up from below by a gas torch with the maximum heating power of $3.5 \mathrm{kWt}$ ). The scheme of the experimental unit and heating modes are described in details in $[5,6]$. To visualize the vortex structures formed were used mcm sized particles of magnesia $\left(4 \mathrm{MgCO}_{2} \mathrm{Mg}(\mathrm{OH}) 24 \mathrm{H}_{2} \mathrm{O}\right.$, physical density of $\left.3900 \mathrm{~kg} / \mathrm{m}^{3}\right)$, which were thinned on the substrate sheet before starting the experiments. The scheme of the experimental unit is presented in Fig. 1.

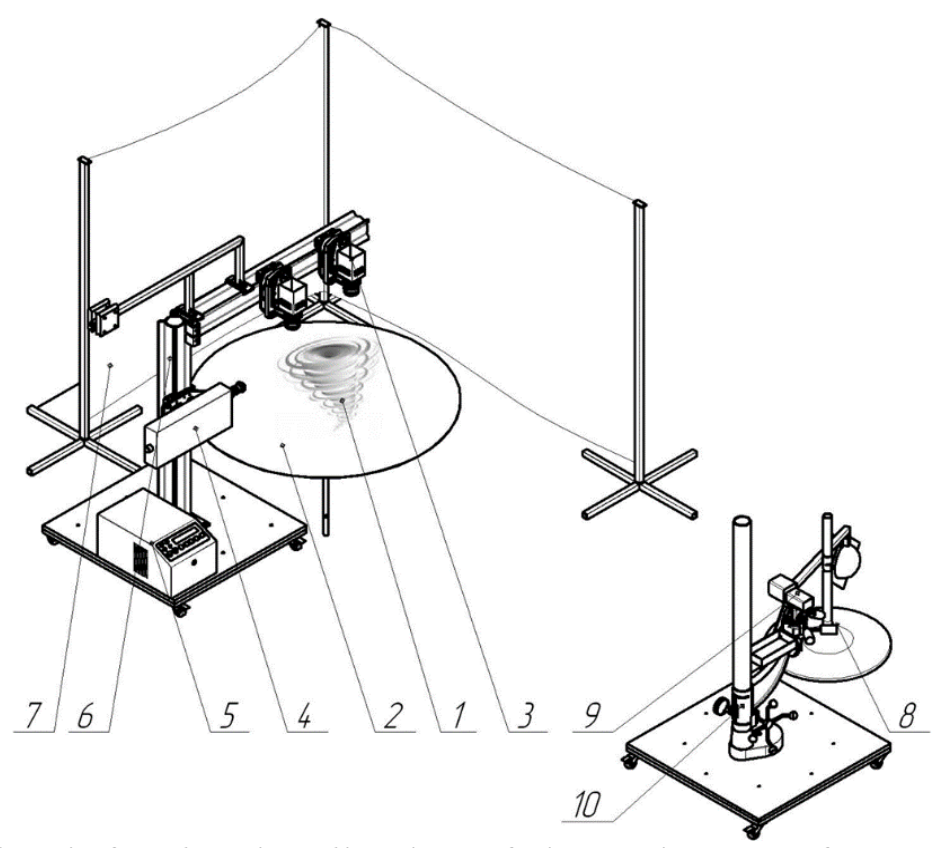

Fig. 1. Experimental unit for the visualization of air vortices: 1 - free non-stationary vortex; 2 - substrate surface; 3 - digital photo camera; 4 - laser; 5 - synchronization unit; 6 frame; 7 - background screen; 8 - illumination console; 9 - digital video camera; 10 supporting rack

Two methods are used to observe and register the vortices. The former is the direct video registration (filming) with use of a digital video camera (here Sanyo VCC-6572P, Japan). The latter is the PIV method (performed by the use of the measurer of fields of velocities POLIS (Russian "ПОЛИС", the instrument is developed by the Institute for Thermophysics of the Siberian Branch of RAS, Novosibirsk). 


\section{Visualization of free non-stationary vortices by the di- rect video registration}

To perform the direct video registration are used several LED floodlights. This approach, due to the light scattering by particles, enables a better visualization of a free nonstationary air vortex. The main problem of these experiments is the uncertainty of the vortex appearance location and time moment. After its appearance a vortex does not remain immobile but moves along a stochastic trajectory, and the vortex structure is nonstationary as well.

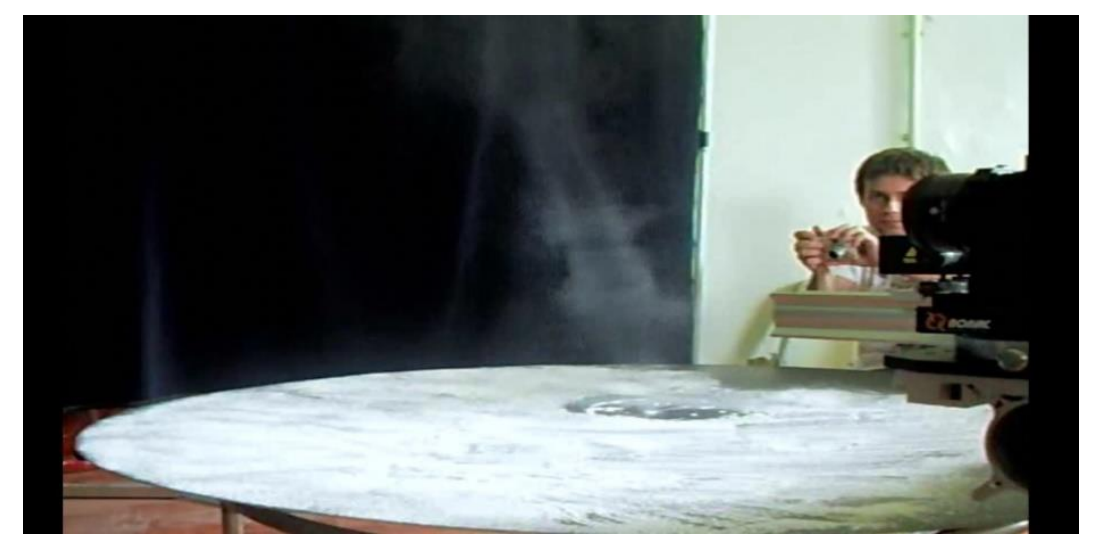

Video 1. Video registration of a free non-stationary vortex

The frame-by-frame analysis of video recordings at various thermal modes allows obtaining the information on the following parameters and characteristics of the vortices generation process: 1) temperatures at which the vortices generation occurs; 2) area of the substrate surface where vortices are formed; 3) direction of the vortex structure rotation; 4) quantity of vortices observed per one experimient; 5) trajectory of motion of the vortex structure base; 6) trajectory length of the vortex base motion; 7) velocity of motion the vortex base; 8) vortex structure lifetime; 9) visible vortices height; 10) visible diameter of vortices etc.

In video 1 there is a periodical appearance of particles highlighted by the green laser plate. These are the particles from the motion pattern of which is performed the vector field building by the PIV method.

\section{Vector fields of instantaneous velocities obtained by the PIV method}

Measurements of fields of velocities based upon the observation of small tracer particles entrained by a flow are known within several tens of years. But the previous manual data processing was extremely difficult and, due to this, the quality and amount of information necessary for the solution of physical tasks was not accessible.

The PIV method is one of the optical techniques to measure velocities of single- [7-9] and two-phase [10] flows. As compared with other methods of flow structure investigation it is of special importance due to its ability to register the instantaneous spatial distributions of velocities. This advantage is very essential for studying the turbulent flows having large vortex structures. When using single-point methods of diagnosis the information on the characteristics of these structures can be lost. But using of PIV and similar methods allows obtaining the information on the dynamics of vortex structures, their dimensions, differential characteristics calculation, time-spatial correlations and static flow characteristics as well [11-14].

The measurement of instantaneous flow velocity fields is based upon the measurement of the movement of admixed particles in the cross-section area plane within a fixed time in- 
terval. In a liquid or gas flow are admixed small particles (tracers) whose size, density and volumetric concentration are chosen to minimize effects of two-phase character of flow and particles buoyancy. The measurement part of a flow is that one "cutted of" by the light blade. The particles images are registered by a film or digital camera.

Basements of image processing. The essence of the PIV method consists in the processing of images obtained. There are several modifications of the tracer particles visualization and respectively various processing techniques. Two types of correlation algorithms are distinguished: in the auto-correlation technique both the initial and final position of tracer particles are registered onto one and the same frame and the cross-correlation one when they are registered on different frames. Using of the latter is more preferable (though it requires more resources), since in autocorrelation algorithms initial and final positions are equivalent, and, respectively, their shift is determined up to a sign. In this case the a priori knowledge about the flux structure is required. The whole measurement area is broken up into elementary cells (calculation areas) with the dimensions of $d_{x} \times d_{y}$. The intensity of the light reflected by particles and registered by the camera (grade of grey) can be represented as a function of two variables $x$ and $y: I_{1}(x, y)$ and $I_{2}(x, y)$ for the first and second frame, respectively. Then the following correlation function is calculated:

$\varphi_{I_{1} I_{2}}(m, n)=\sum_{l=-d_{y} / 2}^{d_{y} / 2} \sum_{k=-d_{x} / 2}^{d_{x} / 2} I_{1}(k, l) \cdot I_{2}(k+m, l+n)$

Its maximum corresponds to the most probable shift of particles within the elementary area of measurements. The flow velocity is assumed to be uniform within the elementary area, and the movement of all the particles is one of the same.

For more exact determination of the coordinates of the correlation function maximum in its vicinity, we use the interpolation of the correlation function at a sub-pixel accuracy. The coordinate of the maximum is considered to be that one of the maximum of the interpolation function. The form of the interpolation function is the subject of the method improvement.

To calculate the correlation function, the most of PIV method modifications use the standard algorithm of the fast Fourier transformation. To diminish the effect of the finiteness of the elementary volume at the Fourier transformation, is used the standard windows imposition technique. Moreover, using of windows diminishes the contribution into the correlation function for particles adjacent to the boundaries of the elementary area. The latter are highly likely not to be present in the same elementary area of the second image (the socalled effect of the "loosing of pair" and, hence, are the source of the "correlation noise».

When calculating the most likely movement of particles $D$ in a given elementary area, one can obtain the flow velocity in the point, corresponding to the center of the elementary measurement area ( $\Delta t$ - is the specified time delay between two illuminating flashpoints) as follows

$V=S \frac{D}{\Delta t}$

where $S$ is the scaling coefficient. Making these procedures for each elementary area, into which the image is broken up (regularly of irregularly), we can find the field of velocities for the whole area.

The measured two-component values of vectors are projection of the real $(3 \mathrm{D})$ vectors onto the plane perpendicular to the optical axis of the apparatus registering the particles images. To measure the three velocity components are used, as a rule, two register modules with the optical axes oriented against each other at a certain angle.

To study in details the fields of velocities in concentrated vortices, we used the mentioned above instrument POLIS («ПОЛИС»). The latter allows performing measurements of $3 \mathrm{D}$ 
fields of instantaneous velocities in essentially non-stationary flows with the complicated geometry, at a high time-spatial resolution and low inaccuracies. The system POLIS («ПОЛИС») contains the double pulse Nd-YaG laser (wavelength 532.05 nm, pulse power no less than $5 \cdot 10^{6} \mathrm{Wt}$, pulse duration no more than $5 \mathrm{~ns}$, pulse repetition rate 1-8 $\mathrm{Hz}$ ); laser controller and power supply; two digital 4 MPix cameras with the image format $2048 \times 2048$ (pixel size $7.4 \times 7.4 \mathrm{mcm}$, CCD matrix size of $15.15 \times 15.15 \mathrm{~mm}$, vertical refresh frequency of $3.4 \mathrm{~Hz}$, exposure time $120 \mathrm{~ms}$ ); laser synchronizer and photo cameras. The measurements and data processing were performed by a PC with the data acquisition and processing system, which includes two camera controllers and software ActualFlow also developed by the S.S. Kutateladze Institute for Thermophysics of the Siberian Branch of $R A S$.

The double pulse laser. The double pulse laser is a source of the coherent light pulses. It is designed for the fixation of rapid processes in an environment scattering its radiation and registration of the environment changes within a short time interval The laser generates two pulses of coherent light with the high intensity $\left(5 \cdot 10^{6} \mathrm{Wt}\right)$ and low $\left(10^{-8} \mathrm{~s}\right)$ duration. The pulses are irradiated at a time delay against each other. The latter is varied within a wide range $\left(10^{-7}-10^{-1} \mathrm{c}\right)$ and controlled at a high $\left(10^{-8}\right)$ accuracy.

The laser consists of the laser head and chassis. The laser head contains 2 laser resonators, 2 quantifiers with active material- Nd:YAG and pump lamp, beam convergence system, frequency converter of the laser radiation and optical attenuator. The chassis consists of the pump lamps power supply, cooling down and control systems. The laser head and chassis are connected by a flexible hose having power and control cables and cooling down hoses.

To adjust the laser beam thickness, an optical attachment was used. The adjustment was performed by the rotation of the ring in the central part of this optical attachment. Actually, the central ring rotation varies the optical system focal distance within the range of 0.3 M $-\infty$ at an appropriate «laser knife» thickness variation from 0.5 to $3 \mathrm{~mm}$. At larger distances from the laser head to the measurements area the transversal size of laser beam becomes even greater due to its divergence. The beam thickness was measured by the exposure of the photographic paper.

Digital photo cameras. The cross-correlation cameras are designed for two-times registration of tracer particles flow patterns both in a one-frame and two-frame modes. In the latter the time delay between laser flashpoints was varied from $10 \mathrm{mcs}$ to $259 \mathrm{~ms}$.

The photo cameras were mounted on the coordinate devises having two rotation gears per each camera and one mechanism to move cameras against the objective lens (Fig. 2). The first coordinate rotation gear is designed for the rotation of the principal optical axis of a camera. The angle should be adjusted to enable obtaining the image from the area to be measured. The position of the coordinate mechanism is fixed by the screw on the lower limb. The second coordinate mechanism allows varying the angle between the matrix plane and principal optical axis what, in turn, allows obtaining the sharpness within the whole image field. To make a coarse adjustment of this angle one should release the washer under the limb, rotate the mechanism up to the angle desired and tighten the washer. To make a coarse angle adjustment, a washer above the limb is used. This washer is connected with the rotation gear via an eccentric what allows a fine angle setting. The third coordinate axis allows the measurement of the distance between the matrix and objective lens, i.e. enables the adjustment of the effective optical system magnification. Actually, the variation of this distance is equivalent to the using of replaceable rings for the objective lens. If the limb coordinate is set to zero the distance between the matrix and objective lens is equal to the rated value. Is the matrix is moved away from the objective lens, the optical system magnification becomes greater. Therefore, this coordinate mechanism allows obtaining an arbitrary optical system magnification which is no lower than the rated value. However, we should understand that in this case the range of distances between the object 
and camera, at which the object is sharp, is also varied This means that moving the object from the matrix to a certain range makes it "infinitely removed", and the adjustment of the sharpness becomes impossible. Therefore, we had to find a compromise solution between the optical system magnification and the range of distances between the camera and measurement plane where the area to be measured can be seen at an appropriate sharpness.



Fig. 2. Digital photo camera and the coordinate device of the POLIS( «ПОЛИС») system.

Synchronizing processor. The synchronizing processor is designed for the synchronization of the laser and cameras operation, calculation of the necessary time intervals and generation of synchronizing pulses with the set frequency. It has eight identical channels calculating the time intervals with an output on the front panel for each channel. The front panel has an input for the external synchronizing pulse and LED indicators. On the rear panel of the device is an RS232 interface to connect it with the controlling PC.

The results. The results of the of the measurement data processing of air non-stationary vortices obtained by PIV method are shown in video 2.

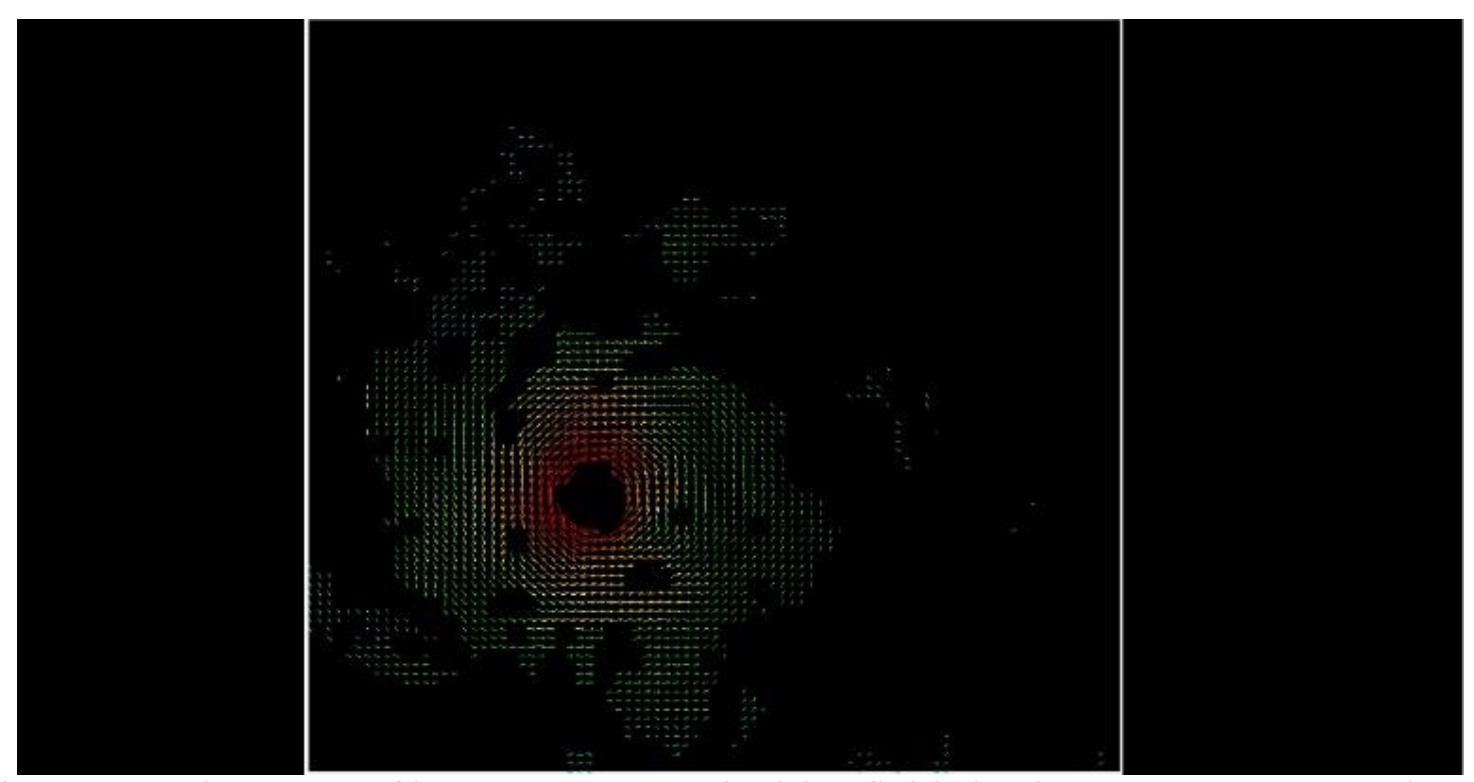

Video 2. Development of instantaneous velocities fields in the vortex transversal crosssection area.

The selective photos with a fixed air vortex at various time moments were processed to find the distribution of velocities over the horizontal plane. The fields of velocities in the model vortex cross-section area are given in Fig. 3 and Fig. 4. 


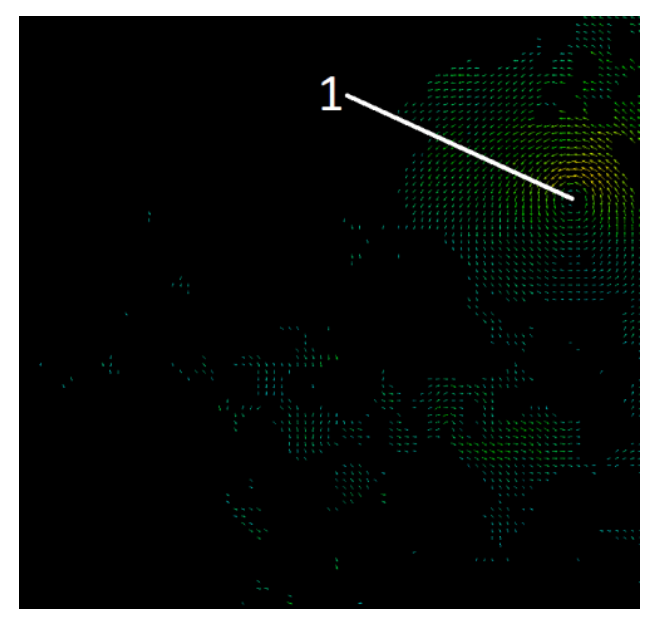

(a)

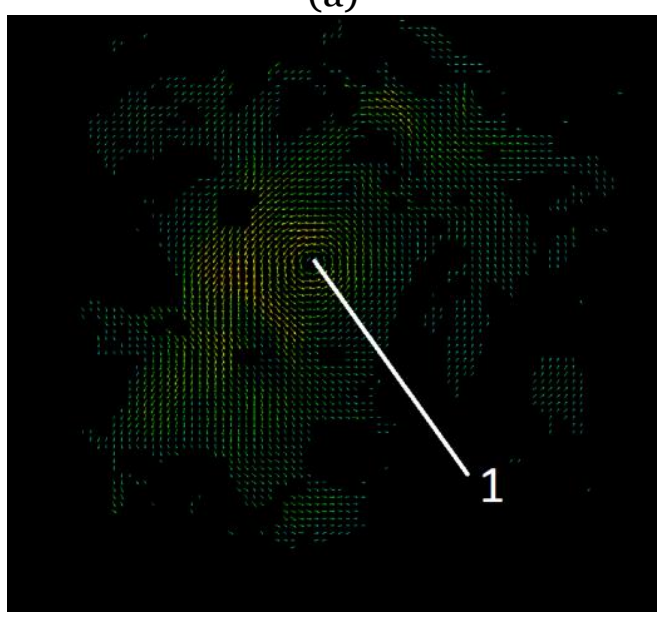

(b)



(c)

Fig. 3. Instantaneous fields of velocities: a) $\tau=3 \mathrm{~s}$, b) $\tau=7 \mathrm{~s}$, c) $\tau=22 \mathrm{~s}$;

1 - non-stationary air vortex; 2 - formed «eye-shaped» vortex. 


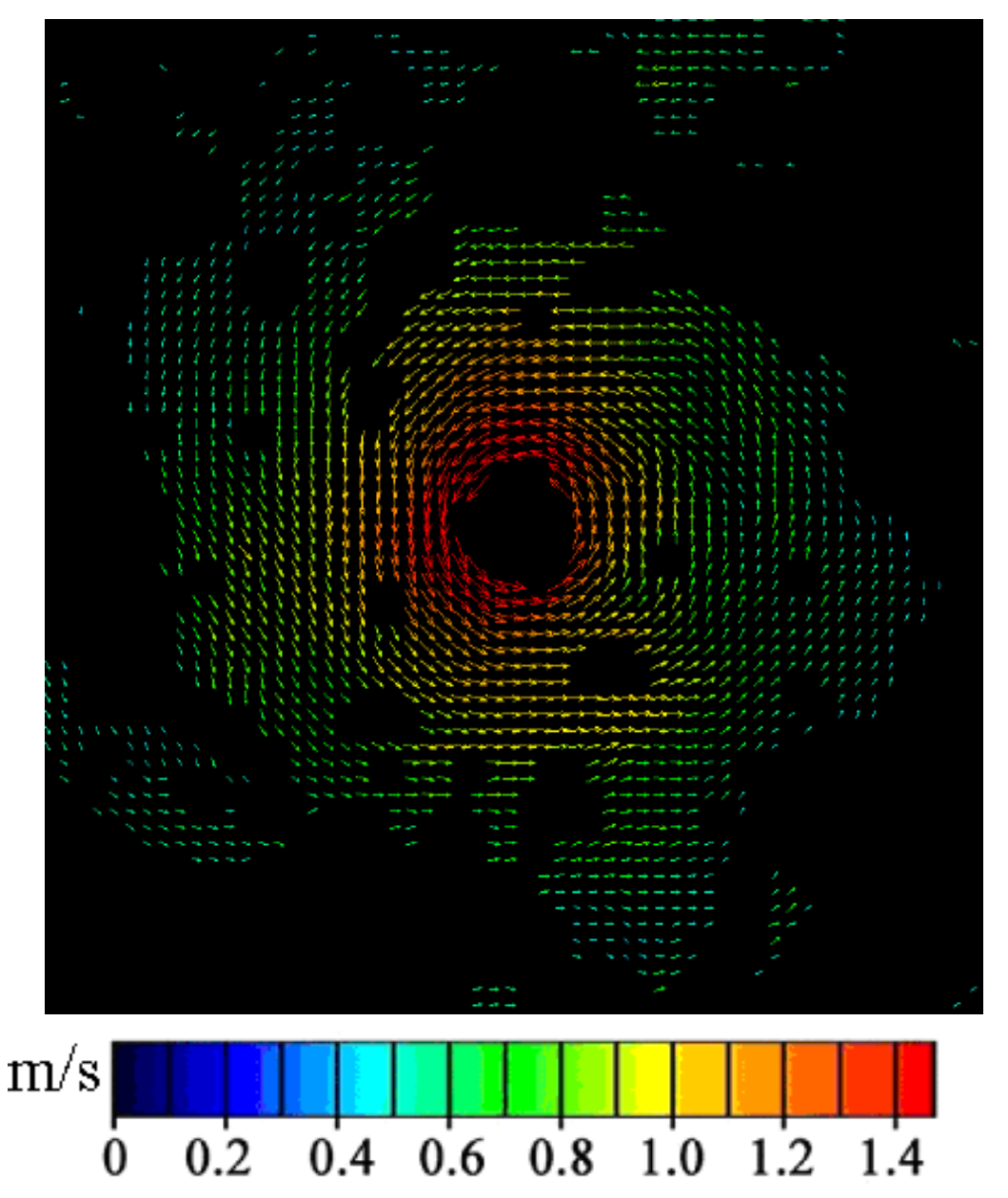

Fig. 4. Instantaneous field of velocities at the moment of maximum development of nonstationary air vortex $(\tau=15 \mathrm{c})$.

To perform a more detailed analysis of the distributions of velocities in the transversal cross-section area of the vortex cavity (see Fig. 4), we consider the scaled up instantaneous field of velocities at the moment of maximum development of non-stationary air vortex structure.

The array of experimental data obtained, concerned with the field of velocities of the vortex structures generated allowed making a conclusion that this distribution of velocities in the vortex cavity is close to that one existing in the classical Rankin vortex.

\section{Conclusion}

In this paper we present the results visualization of free non-stationary vortices by direct video filming and vector fields of instantaneous velocities in the vortex cross-section area obtained by the PIV method.

The developed technique of visualization and diagnosis of free non-stationary vortices can be used for the development of cooling systems for high-voltage megawatt range electric power devices.

\section{Acknowledgement}

This work was supported by the Ministry of Education and Science of the Russian Federation (contract № 14.604.21.0178, project identificator RFMEFI60417X0178). 


\section{References}

1. Wang P., McCluskey P., Bar-Cohen A. Two-Phase Liquid Cooling for Thermal Management of IGBT Power Electronic Module. Journal of Electronic Packaging, 2013, vol. 135, № 2, 021001.

2. Bar-Cohen A., Matin K., Narumanchi S. Nanothermal Interface Materials: Technology Review and Recent Results. Journal of Electronic Packaging, 2015, vol. 137, № 4, 040803 .

3. Bar-Cohen A. Gen 3 "Embedded Cooling": Key Enabler for Energy Efficient Data Centers. IEEE Transactions on Component Packaging and Manufacturing Technology, 2017, vol. 7, № 8, p. 1206-1211.

4. Zeigarnik Y.A., Vasil `ev N.V., Druzhinin E.A. Kalmykov I.V., Kosoi A.S., Khodakov K.A. Prospects for Boiling of Subcooled Dielectric Liquids for Supercomputer Cooling. Doklady Physics, 2018, vol. 63, № 2, p. 58-60.

5. Varaksin A.Y., Romash M.E., Kopeitsev V.N., Gorbachev M.A. Experimental Study of Wall-Free Non-Stationary Vortices Generation due to Air Unstable Stratification. Int. J. Heat Mass Transfer, 2012, vol. 55, p. 6567-6572.

6. Varaksin A.Yu., Romash M.E., Kopeitsev V.N. Tornado. New York: Begell House, 2015.

7. Adrian R.J. Particle Imaging Techniques for Experimental Fluid Mechanics. Ann. Rev. Fluid Mech, 1991, vol. 23, p. 261-304.

8. Adrian R.J. Bibliography of Particle Velocimetry Using Imaging Methods: 19171995. TSI Inc., Minnesota, 1996.

9. Raffel M., Willert C.E., Wereley S.T., Kompenhans J. Particle Image Velocimetry. Springer, 2007.

10. Varaksin A.Yu. Collisions in Particle-Laden Gas Flows. New York: Begell House, 2013.

11. Skornyakova N.M., Sychev D.G., Varaksin A.Yu., Romash M.E. Vizualization of Vortex Structures by Particle Image Velocimetry. Scientific Visualization, 2015, vol. 7, № 3, p. $15-24$.

12. Shestakov M.V., Tokarev M.P., Markovich D.M.. Application of Time-Resolved Tomographic PIV Technique for the Investigation of Processes Forming of Vortex Structure in Quasi Two-Dimensional Turbulent Jet. Scientific Visualization, 2015, vol. 7, № 3, p. $1-8$.

13. Alekseenko S.V., Dulin V.M., Tokarev M.P., Markovich D.M. A Swirling Jet with Vortex Breakdown: Three-Dimensional Coherent Structures. Thermophysics and Aeromechanics. 2016, vol. 23, № 2, p. 301-304.

14. Shestakov M.V., Mullyadzhanov R.I., Tokarev M.P., Markovich D.M. Modulation of Large-Scale Meandering and Three-Dimensional Flows in Turbulent Slot Jets. Journal of Engineering Thermophysics. 2016, vol. 25, № 2, p. 159-165.

15. Alekseenko S.V., Abdurakipov S.S., Hrebtov M.Y., Tokarev M.P., Dulin V.M., Markovich D.M. Coherent Structures in the Near-Field of Swirling Turbulent Jets: A Tomographic PIV Study. International Journal of Heat and Fluid Flow. 2018, vol. 70, p. 363-379. 